\title{
Approche spatiale de la conversion à l'agriculture biologique. Les dynamiques régionales en France
}

Spatial approach on conversion to organic farming: regional dynamics in France

Gilles Allaire, Éric Cahuzac, Thomas Poméon et Michel Simioni

\section{(2) OpenEdition}

\section{Journals}

\section{Édition électronique}

URL : http://journals.openedition.org/economierurale/4200

DOI : 10.4000/economierurale.4200

ISSN : 2105-2581

\section{Éditeur}

Société Française d'Économie Rurale (SFER)

\section{Édition imprimée}

Date de publication : 20 janvier 2014

Pagination : 9-31

ISSN : 0013-0559

\section{Référence électronique}

Gilles Allaire, Éric Cahuzac, Thomas Poméon et Michel Simioni, « Approche spatiale de la conversion à l'agriculture biologique. Les dynamiques régionales en France », Économie rurale [En ligne], 339-340 | janvier-mars 2014, mis en ligne le 20 janvier 2016, consulté le 19 avril 2019. URL : http:// journals.openedition.org/economierurale/4200 ; DOI : 10.4000/economierurale.4200 


\title{
Approche spatiale de la conversion à l'agriculture biologique Les dynamiques régionales en France
}

\author{
Gilles ALLAIRE, Éric CAHUZAC, Thomas POMÉON • Observatoire des Programmes Communautaires \\ de Développement Rural, US INRA-SAE2 \\ Michel SIMIONI • Toulouse School of Economics, GREMAQ-INRA-IDEI
}

Les auteurs proposent dans cet article une analyse spatiale exploratoire (ESDA) de la conversion à I'agriculture biologique (AB) en France depuis 1994. Ils mobilisent pour cela les données individuelles sur les aides versées aux agriculteurs pour la conversion à l'AB (CAB) dans ses différentes mises en œuvre historiques. Ils construisent tout d'abord un jeu long de données (1994-2009) sur des microterritoires, à partir duquel les auteurs mettent en évidence des typologies régionales et locales d'évolution de l'effort de contractualisation des aides $C A B$ en termes d'intensité, de concentration, d'agglomération et de contagion. Parmi les principaux résultats, on note que si la diffusion de $I^{\prime} A B$ présente encore un caractère hétérogène aux niveaux inter- et intra-régional, la tendance générale est à la réduction de la disparité entre territoires. Des pistes d'explication sont entrevues, qui requièrent une analyse plus ciblée pour pouvoir être validées.

MOTS-CLÉS : ESDA, agriculture biologique, politique du développement rural, indices de concentration, moran, territoires, dépendance spatiale

\section{Spatial approach on conversion to organic farming: regional dynamics in France}

We propose in this article an exploratory spatial analysis (ESDA) of conversion to organic farming (OF) in France since 1994. We mobilize individual data on support to farmers for conversion to OF, in its various historical implementations. First we build a longitudinal data set (1994-2009) on micro-territories, from which we highlight different regional and local typologies of OF support contracting evolution, in terms of intensity, concentration, agglomeration and contagion. Among the main results, we note that if the distribution of OF still has a heterogeneous pattern interand intra-regional, the general trend is towards the reduction of disparity between territories. Some possible explanations are foreseen, which require a more detailed analysis to be validated. (JEL: C11, C21, Q01, Q18).

KEYWORDS: ESDA, organic farming, rural development policy, concentration indices, moran, territories, spatial dependence

$\mathbf{L}$ a France est un des pays européens où l'agriculture biologique (AB) est la moins développée. Néanmoins, elle tend à rattraper le retard pris dans la dernière décennie, passant du $21^{\mathrm{e}}$ rang de $1^{\prime}$ 'UE en 2009 au $19^{\mathrm{e}}$ rang fin 2010 quant à la part de la Surface agricole utilisée (SAU) bio dans la SAU nationale (Agence Bio, 2012); soit $2,5 \%$ de la SAU pour 2009 et 3,1\% pour 2010. Toutefois, le développement de l'AB reste en deçà des attentes politiques. Il s'avère donc utile de mieux comprendre les facteurs socio-économiques favorisant

ou freinant la diffusion de l'AB, que ce soit au niveau des unités de production, des filières ou encore des territoires. La présente recherche concerne essentiellement la diffusion territoriale de l'AB.

La question de l'adoption des méthodes de production de l'AB a été abordée dans de nombreux travaux ${ }^{1}$. Des opportunités économiques, des capacités et des

1. Pour un bilan de la littérature en sciences sociales sur la conversion à l'AB voir Géniaux et al. (2010). 


\section{Approche spatiale de la conversion à l'agriculture biologique}

motivations personnelles constituent les déterminants de l'adoption généralement cités. Des caractéristiques personnelles et celles de l'exploitation agricole (dont sa localisation) entrent en ligne de compte. Les travaux sur l'adoption de l'AB reposent nécessairement sur des enquêtes auprès des producteurs. La principale source de données utilisée dans ce travail, qui est de nature administrative, ne permet pas d'apporter des éléments nouveaux pour l'étude de ces déterminants, car elle contient très peu d'information sur les exploitations concernées. Par contre, il s'agit d'une source exhaustive qui recense les surfaces converties en $\mathrm{AB}$ avec des aides publiques, en France, depuis 1994, et qui permet de ce fait d'analyser les mécanismes de diffusion spatiale de l'AB au cours du temps. Il faut distinguer les déterminants de l'adoption, qui est une décision individuelle, de ceux de la diffusion résultant de l'agrégation des comportements d'adoption. Différents facteurs interviennent alors, qui sont des facteurs de localisation : accès aux marchés et circuits de commercialisation, réseaux sociaux et actions professionnelles, politiques de soutien régionales ou locales, etc. L'ambition de ce texte n'est pas de spécifier et hiérarchiser ces déterminants, mais plutôt de montrer qu'il y a bien une structuration spatiale à différentes échelles de la diffusion de l'agriculture biologique.

La répartition spatiale de l'AB en France présente des contrastes importants. La majorité des communes ne compte aucune exploitation en agriculture biologique et celles qui en comptent ne sont pas uniformément réparties. La moitié des surfaces consacrées à l'AB se concentre dans cinq régions (qui ne totalisent que $30 \%$ de la SAU française métropolitaine) (Agence Bio, 2012). Parce que les régions interviennent par des politiques propres et dans la mise en œuvre des politiques publiques nationales et européennes en soutien au développement de l'AB, nous privilégions ce niveau d'analyse et nous proposons dans ce texte une analyse comparative du développement et des formes de répartition spatiale de l'AB dans les régions françaises au cours des deux dernières décennies.

Nous utilisons les méthodes de l'Exploratory Spatial Data Analysis (ESDA) (Anselin, 2005) pour étudier quatre aspects de la diffusion de l'AB : intensité, concentration, agglomération et contagion. Pour chaque unité spatiale élémentaire (l'échelle utilisée ici se situe entre commune et canton), nous connaissons la surface convertie en $\mathrm{AB}$, pour quatre périodes correspondant à différentes phases des politiques publiques de soutien à la conversion à l'agriculture biologique. L'intensité et la concentration peuvent se mesurer à différentes échelles. L'agglomération et la contagion désignent les phénomènes de répartition des unités spatiales où se concentre l'AB versus où elle est faiblement ou pas représentée.

L'intérêt d'étudier les effets d'agglomération de la localisation des cultures biologiques est multiple, tant du point de vue des impacts environnementaux de ce mode de production que de l'organisation des filières $\mathrm{AB}$. Du point de vue de la biodiversité, il est généralement considéré que l'optimum est une répartition de pôles de surfaces exploitées en $\mathrm{AB}$; mais à notre connaissance il n'y a pas de travaux permettant de définir une taille optimale pour ces pôles et la maille de répartition nécessaire pour assurer un impact effectif. Du point de vue économique, la répartition spatiale peut notamment s'interpréter à partir de la question de l'écoulement de la production. La répartition des marchés locaux et des points ou circuits de collecte des produits (lait, céréales) de l'AB, ainsi que la proximité d'une clientèle pour la vente directe et en circuits courts conditionnent la localisation de la production. Enfin, du point de vue sociologique, l'existence de réseaux et de leaders locaux stimule la conversion à l’AB. 
Plusieurs études, à différentes échelles et dans différents pays ${ }^{2}$, se sont intéressées à la diffusion de l'AB, à partir d'indices de concentration (Beauchesne et Bryant, 1999 ; Ilbery et al., 1999 ; Risgaard et al., 2007 ; Ilbery et Maye, 2011) ou en se concentrant sur les effets d'agglomération et de voisinage (Nyblom et al., 2003 ; Bichler et al., 2005 ; Gabriel et al., 2009 ; Frederiksen et Langer, 2004 ; Lewis et al., 2011). Peu ont cherché à intégrer une dimension temporelle (Nyblom et al., 2003 ; Lewis et al., 2011). Il en ressort qu'en fonction de l'échelle considérée, les résultats diffèrent en termes de significativité et de tendance. Il faut donc rester prudent sur la portée des résultats face à la complexité des causalités qui sont en jeu dans la localisation de l'AB. Dans le cas français, les travaux de Géniaux et al. (2009) sur la Région Provence-AlpesCôte d'Azur ont constitué une première approche de la diffusion de l'AB depuis une perspective spatiale. L'originalité du travail présenté ici est de proposer une analyse comparative à l'échelle des régions françaises intégrant la dimension temporelle.

Après avoir présenté la dynamique générale de l'AB en France et les données mobilisées, nous construirons des types d'évolution au niveau régional faisant apparaître l'hétérogénéité en termes de diffusion et de concentration de l'AB. L'analyse reposera ensuite sur des techniques d'analyse spatiales pour déterminer les modalités régionales de la concentration des surfaces converties au cours des différentes périodes. Nous distinguerons ainsi les cas où l' $\mathrm{AB}$ se concentre dans quelques microterritoires répartis sur l'ensemble de la région de ceux où l' $\mathrm{AB}$ se développe dans des grappes agglomérées

2. Ces études portent sur l'Angleterre et le Pays de Galles, le Danemark, la Finlande, les États-Unis, le Canada, l'Allemagne, sur tout ou partie du territoire national. de microterritoires. Cela nous conduira à la recherche de corrélations spatiales et temporelles dans cette diffusion et à émettre quelques propositions pour leur interprétation.

\section{La conversion à l'agriculture biologique}

\section{Des variations régionales}

Les taux de SAU convertie ou en conversion $\mathrm{AB}$ varient selon les régions de 0,7 à 12,3\% en 2011 (Agence Bio, 2012). Les régions varient également, comme nous allons le voir, selon les rythmes d'évolution et les formes de concentration de l'AB.

L'étude conduite par l'ASP (Quelin, 2010) met en évidence deux importants facteurs de localisation régionale de l'AB, l'un tenant aux systèmes de production dominants, l'autre aux crédits d'animation attribués aux structures d'information, d'animation et de conseil (enveloppes régionales attribuées des plans nationaux de développement de l'AB). Par ailleurs, l'incitation financière à la conversion est d'autant plus efficace que, d'une part, l'organisation des filières concernées réduit les risques liés à la recherche de débouchés et, d'autre part, que le montant de l'aide publique couvre les coûts de conversion. Or, dans le dispositif des aides à la conversion $(\mathrm{CAB})^{3}$, le montant de la compensation est différent selon le type de production et, d'après le rapport

3. La CAB permet d'aider l'exploitant agricole durant la période de transition entre l'agriculture conventionnelle et l'agriculture biologique. Selon le principe des Mesures agro-environnementales (MAE), elle vise à compenser la perte de revenu engendrée lors de la conversion tandis qu'un écoulement de la production sous le label AB n'est pas possible. Cette aide est surfacique et concerne donc à la fois des exploitations nouvellement converties (totalement ou partiellement) et des anciennes qui s'agrandissent. 
de l'évaluation finale du PDRN ${ }^{4}$ (AND-I, 2008), l'aide ne compensait réellement les pertes et manques à gagner de la période de conversion que pour les prairies à faible chargement et les vergers en fruits secs. On peut donc dire, comme Quelin (2010), que, depuis 2000, la $\mathrm{CAB}$, bien qu'identique sur tout le territoire, a participé à l'hétérogénéité régionale du développement de l'AB, vu l'hétérogénéité spatiale des productions favorisées ou défavorisées par le niveau des aides.

\section{Une dynamique interrompue qui repart}

Les sources d'information sur la conversion à l'agriculture biologique sont l'Agence Bio pour les surfaces en conversion du point de vue réglementaire et l'Observatoire des programmes de développement rural (ODR) pour les aides à la conversion ${ }^{4}$. L'Agence Bio publie des statistiques annuelles sur le nombre d'opérateurs certifiés en $A B$ et les surfaces concernées (certifiées $\mathrm{AB}$ ou en conversion), pour trois niveaux géographiques : national, régional et départemental. Dans le cadre de l'ODR, sont disponibles des données individuelles anonymes fournies par l'ASP sur les CAB, localisées à la commune.

Si selon les régions françaises l'importance de la $\mathrm{AB}$ varie, la conversion à l' $\mathrm{AB}$ est également un phénomène qui varie dans le temps (cf. le graphique 1 qui utilise les deux sources Agence Bio et ODR). Une phase de forte progression des surfaces en conversion depuis 2006 a succédé à une période de diminution (2003-2005) ; elle affecte la quasi-totalité des régions françaises. Cette progression s'est consolidée, avec un quasi-doublement des surfaces en conversion entre 2008 et $2009(+86 \%)$ et entre 2009 et $2010(+80 \%)$, dans une dynamique comparable à celle connue en 1998-1999. Des politiques publiques plus incitatives, incluant notamment le déplafonnement des aides $\mathrm{CAB}$ et un accès plus

Graphique 1. Évolution de l'agriculture biologique et des aides à la conversion en France sur la période 1995-2010

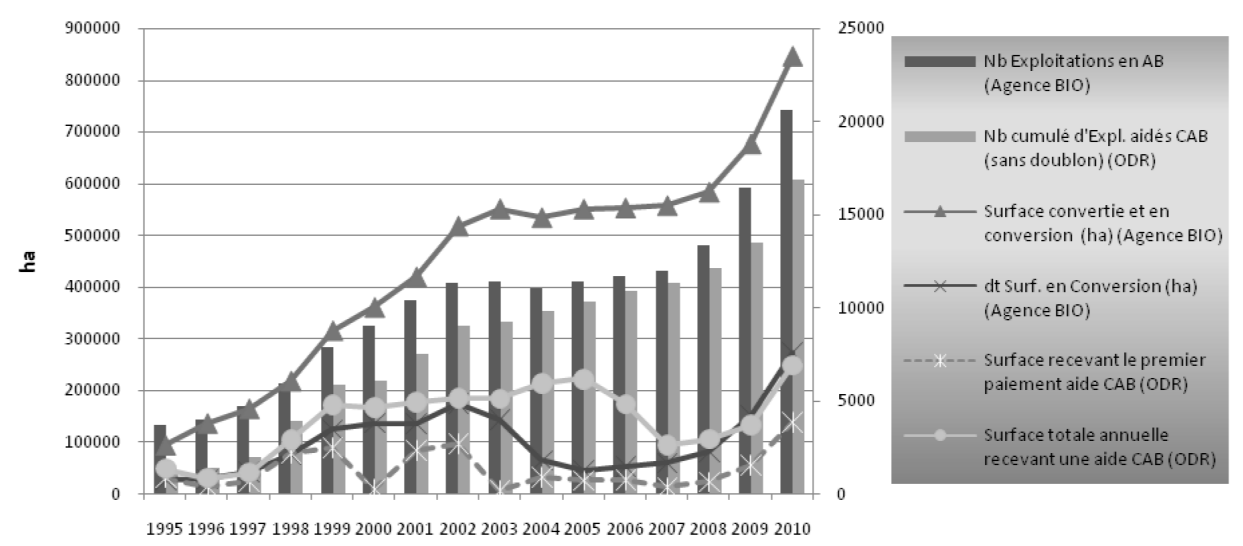

Source : Agence Bio 2012 / ODR.

4. Pour rappel, selon la réglementation, la conversion d'une parcelle se fait sur une durée de 2 ans pour des cultures annuelles et les prairies, et de 3 ans pour les cultures pérennes. La prime à la conversion est payée pendant 5 ans et l'engagement de l'agriculteur est aussi de 5 ans. 
RECHERCHES

avantageux au crédit d'impôt, ont accompagné cette hausse.

Pour obtenir une aide à la conversion (CAB), la certification est nécessaire. Une partie des bénéficiaires de l'aide arrête la certification à la fin de la durée de l'engagement de cinq ans, sans d'ailleurs abandonner complètement les méthodes de l'AB, mais en s'accordant plus de flexibilité face à des risques majeurs. Néanmoins, ces comportements, dont il existe des témoignages, mais qui sont mal connus, sont limités ; les estimations que nous avons pu faire à partir de la comparaison des sources de l'Agence Bio et de l'ASP laissent penser que plus de $80 \%$ des exploitations en $\mathrm{AB}$ ont reçu des aides pour la conversion. La diffusion des $C A B$ que nous étudions dans cet article est donc fortement représentative de la diffusion de l'agriculture biologique.

\section{Les dispositifs d'aide à la conversion dans la PAC}

L'Union européenne dispose depuis 1991 d'une réglementation pour l'AB et une aide à la conversion à l'agriculture biologique $(\mathrm{CAB})$ a été créée par le règlement européen $n^{\circ}$ 2078/92 pour la période 19931999, reconduite dans les deux règlements de développement rural successifs au titre des Mesures agro-environnementales (MAE). Il s'agit d'aides cofinancées par l'État et par l'Union européenne. Le premier règlement européen de développement rural (2000-2006) s'est traduit en France par le Plan de développement rural national (PDRN), la CAB correspondant à une MAE mise en œuvre dans le cadre des Contrats territoriaux d'exploitation (CTE) puis des Contrats d'agriculture durable (CAD) qui les ont remplacés, avec moins d'ambition et de moyens. Le second règlement (2007-2013), qui a donné lieu au Plan de développement rural hexagonal (PDRH), inclut la CAB ainsi qu'une mesure d'aide à la certification (retenue dans une partie des régions seulement $)^{5}$. Il existe aussi des aides régionales et locales, qui peuvent influencer la localisation de l'AB.

\section{Les données mobilisées}

Nous mobilisons les données individuelles des aides à la conversion, sous les différentes formes qu'elles ont prises depuis leur création (source : $\mathrm{ASP}^{6}$ ). Ces aides, dont le montant à l'hectare varie selon le type de culture, ont concerné la très grande majorité des exploitations converties à l'AB. Ces données concernent la France continentale (la Corse étant exclue du PDRH, elle n'est pas incluse dans l'étude) et portent sur les surfaces converties. Les bénéficiaires sont localisés au niveau communal ${ }^{7}$.

Le tableau 1 indique le nombre d'individus et les surfaces engagées selon les dispositifs publics impliqués et les périodes considérées. Ce jeu de données confirme les tendances d'évolution de l'AB décrites précédemment à partir des données de l'Agence Bio. Les surfaces nouvelles engagées en $\mathrm{CAB}$ ont été particulièrement faibles entre 2003 et 2007, avant de connaître un regain sensible (graphique 1). Dans l'interprétation des valeurs annuelles, il faut prendre en compte les changements de dispositif avec une faible contractualisation les années de changement, soit 2000, 2003 et 2007 ; aussi nous raisonnons ici par périodes.

5. Le lecteur trouvera plus informations sur les aides pour l'AB sur le site de l'Agence Bio (http:// www.agencebio.org).

6. Agence de service et de paiement (ex. : CNASEA), qui est en charge - depuis le début de leur mise en place - du contrôle des dossiers de demande d'aides $\mathrm{CAB}$ et du paiement de ces aides aux agriculteurs.

7. Ces données sont disponibles sur la plate-forme ODR et utilisées ici avec l'autorisation du Comité de pilotage de l'Observatoire des programmes communautaires de développement rural : http:// odr.supagro.inra.fr. 
Approche spatiale de la conversion à l'agriculture biologique

Tableau 1. Nombre d'individus et surfaces ${ }^{8}$ aidées par mesures

\begin{tabular}{lcccccc}
\hline \multicolumn{1}{c}{ Mesures } & & $\begin{array}{c}\text { Avant 2000 } \\
\text { compris }^{9}\end{array}$ & 2001-2003 & 2004-2006 & 2007-2009 & Cumul \\
\hline MAE 2078 & (nb) & 5861 & - & - & - & 5861 \\
\multirow{2}{*}{ CTE } & $(\mathrm{ha})$ & 237452 & - & - & - & 237452 \\
& $(\mathrm{nb})$ & 244 & 3717 & - & - & 3961 \\
CAD & $(\mathrm{ha})$ & 8374 & 185804 & - & - & 194178 \\
& $(\mathrm{nb})$ & - & - & 2007 & 64 & 2071 \\
MAE RDR2 & $(\mathrm{ha})$ & - & - & 84700 & 2257 & 86957 \\
& $(\mathrm{nb})$ & - & - & - & 3129 & 3129 \\
\multirow{2}{*}{ Surfaces totales } & $(\mathrm{ha})$ & - & - & - & 87940 & 87940 \\
\hline
\end{tabular}

Note : Les calculs sont réalisés sur les contrats signés.

Source : CNASEA-ASP/ODR.

La $\mathrm{CAB}$ est une mesure plafonnée par exploitation et qui, dans le cadre du PDRH, ne peut pas être cumulée pour une même parcelle avec une autre aide " surfacique » (par exemple la Prime herbagère agro-environnementale, PHAE) ; les surfaces converties par exploitation peuvent donc être supérieures aux surfaces percevant une $\mathrm{CAB}$. Cette sous-estimation des surfaces converties ne modifie cependant pas le nombre d'exploitations qui se convertissent ni leur localisation, mais peut toutefois induire une légère sous-estimation de l'intensité de la conversion. Ces réserves faites, c'est à partir des données sur les $\mathrm{CAB}$ que nous développerons notre analyse. Dans un premier temps, les données individuelles ont été ramenées à une échelle géographique infra-cantonale,

8. Sont dénombrées les surfaces aidées uniquement pour l'année d'engagement de l'aide ; ceci évite donc les doublons et permet de faire ressortir la dynamique propre à chaque période.

9. Une première série de CTE a été signée en 2000, dans le cadre d'une mise en place accélérée, hors financement européen. Toutefois, afin de garder par la suite une homogénéité temporelle des périodes ( 3 ans), nous avons considéré le début de la seconde période en 2001 . prenant en compte une certaine homogénéité du contexte biophysique, agronomique et institutionnel. Ces zones seront désignées par la suite par le terme de « microterritoire (d'étude) $»^{10}$. Nous allons maintenant présenter les résultats de l'analyse spatiale des aides $\mathrm{CAB}$, débouchant dans un premier temps sur une typologie des régions en fonction des dynamiques de diffusion de l'AB dans l'espace régional.

\section{Analyse spatiale de la conversion à l'agriculture biologique}

Nous étudions, dans un premier temps, la concentration des surfaces aidées en $\mathrm{CAB}$ dans les microterritoires, qui sont

10. Dans cet article, le terme microterritoire renvoie à une simple délimitation géographique. Toutefois, nous avons choisi une méthode de zonage visant à créer des microterritoires qui ont une certaine homogénéité du point de vue agronomique (par l'altitude), de la proximité aux pôles urbains et du point de vue des politiques territoriales. Ce découpage comprend des portions de cantons homogènes selon 3 zonages : celui des aires urbaines et son extension à l'espace rural (ZAUER, 1999), les zones montagne, défavorisées et plaine, et les zones d'objectifs UE 1 ou 2. 
les unités spatiales élémentaires de l'analyse, en calculant un indicateur d'intensité relative de la conversion en référence à la moyenne régionale ; puis nous construisons une typologie des régions quant à la dynamique de croissance et de répartition spatiale de l'AB au cours des quatre périodes considérées dans l'analyse (points 1 et 2). La distribution spatiale de la concentration locale en $\mathrm{AB}$ pouvant recouvrir de multiples configurations, nous recherchons s'il existe des effets d'agglomération de microterritoires avec une forte concentration en $\mathrm{AB}$ et nous proposons une typologie des formes d'agglomération (points 3 et 4). Enfin, nous vérifions l'existence de phénomènes de dépendance spatio-temporelle dans la localisation de l'AB (point 5) ${ }^{11}$. L'analyse statistique conduite vise uniquement la mise à jour des configurations spatiales.

Nous appréhendons la capacité différentielle des microterritoires à abriter et à concentrer des surfaces converties en $\mathrm{AB}$, en tenant compte de la capacité des zones voisines pour cerner les effets d'agglomération. Suivant l'usage en économie, nous utiliserons le terme « effort » (de contractualisation ou de conversion) pour exprimer une plus ou moins grande capacité des territoires à contenir des surfaces en AB. Cet effort est fourni par les agriculteurs qui convertissent leur exploitation et par le «territoire » lui-même, lorsque des collectivités ou des associations locales soutiennent l'effort des premiers. Il est mesuré par le taux de conversion des surfaces agricoles et dépend d'une part des motivations individuelles et des capacités collectives dans un territoire, et d'autre part de la localisation de ce territoire, pour différentes raisons tenant au système de

11. Faute de place, tous les résultats et cartes commentés ici ne sont pas présentés dans l'article. Néanmoins, ils sont disponibles sur demande auprès des auteurs et sur la plate-forme ODR. production en place et de la proximité des territoires débouchés. L'analyse présentée ici ne va pas plus loin dans l'explicitation de cet effort, mais cette conceptualisation est néanmoins nécessaire pour considérer que la localisation de l'AB n'est pas due au seul hasard, et qu'il y a donc un sens à mettre en évidence les configurations spatiales de l'AB.

\section{Intensité relative de la conversion au niveau régional}

La répartition en termes absolus des surfaces converties dans les microterritoires, qui sont de taille variable, ne permet pas d'évaluer l'effort de conversion de chacun. Pour cela, nous calculons un quotient localisé (QL) qui permet de comparer la part relative des surfaces en $\mathrm{AB}$ entre microterritoires appartenant à un même ensemble géographique. Ce quotient est le rapport de deux proportions : la part des surfaces en CAB de la zone dans la SAU du microterritoire et celle en CAB dans la SAU dans l'ensemble géographique considéré. Nous avons choisi de rapporter le calcul du QL aux régions administratives, vu le constat de l'hétérogénéité régionale des taux de conversion et des dispositifs d'appui à la conversion (cf. ci-dessus). Soit :

$$
x_{i r}=\frac{\frac{S_{i}}{S A U_{i}}}{\frac{S_{r}}{S A U_{r}}}, \quad \forall i, r
$$

avec $S_{i}$ et $S_{\mathrm{r}}$ les surfaces engagées en CAB respectivement sur le microterritoire $(i)$ et la région $(r)$ et SAU représentant les surfaces agricoles utilisées à ces mêmes niveaux géographiques. Une valeur supérieure à 1 de $x_{i r}(\mathrm{QL})$ signifie que la part des surfaces en conversion dans le microterritoire est supérieure à la part observée dans la région. Comme le font remarquer Ilbery et Maye (2011), le quotient localisé est assez sensible aux unités locales à faibles effectifs, raison pour laquelle nous avons choisi des microterritoires 
regroupant plusieurs communes (et comprenant au moins une dizaine d'agriculteurs en 2000).

Les valeurs des QL, calculés pour l'ensemble des périodes, varient entre 0 et 147 . La comparaison des QL pour des microterritoires appartenant à différentes régions doit tenir compte que la valeur du quotient se rapporte à une référence régionale. Pour des comparaisons interrégionales entre types d'espaces, on peut créer une classe autour de la valeur 1 (microterritoires dont l'effort est proche de la moyenne régionale). Nous avons ainsi examiné la répartition des QL en 3 classes $(<0,8$; $0,8-1,2 ;>1,2)$ selon les types de microterritoires pour trois critères : le zonage en aires urbaines étendu au rural de l'INSEE (ZAUER), version de 1999, celui des zones à « handicaps naturels » de la PAC (hautemontagne, montagne, piémont, zones défavorisées, plaine), ces deux zonages intervenant dans la définition des microterritoires pris en compte, ainsi que le zonage des zones « vulnérables » selon la directive nitrates. Dans les 3 cas, un test de Chi2 permet de rejeter l'hypothèse d'indépendance. Pour toutes les périodes, la classe des QL supérieurs à 1,2 est surreprésentée dans les zones rurales, dans les zones de montagne, de piémont et dites " défavorisées » et en dehors des zones dites « vulnérables », qui sont les zones où dominent les systèmes d'élevage intensif et de grandes cultures, peu enclins à la pratique de l'AB.

Environ $20 \%$ des microterritoires ont un quotient supérieur à 1 , avec une proportion minimale de $15 \%$ pour la période
2004-2006 (tableau 2). La carte 1 en annexe représente ces quotients localisés pour la période 2007-2009. Ce qui fait sens sur la carte est la répartition des QL $>1$. Le taux de territoire avec un $\mathrm{QL}>1$, où régionalement se concentrent les conversions, est logiquement d'autant plus élevé que l'est celui des territoires où le $\mathrm{QL}>1$. La concentration résulte à la fois du nombre de microterritoires où sont présentes des $\mathrm{CAB}$ et de plus ou moindre intensité de la conversion dans ceux-ci. Globalement, moins de territoires sont concernés par la conversion à une période, plus est importante la part de ceux qui ont un $\mathrm{QL}>1$, ce que montre le tableau 2. Toutefois, aux différentes périodes, ces deux facteurs de la concentration peuvent se combiner différemment dans les régions, ce que nous allons étudier maintenant.

\section{Concentration de l'effort de conversion et typologie des régions}

Pour comparer les régions du point de vue de la concentration de l'AB, nous construisons pour chacune, à partir des quotients localisés microterritoriaux, un indice de Gini localisé (Guillain et Le Gallo, 2010). Cet indice est calculé à partir de la somme des écarts entre les QL (en valeur absolue). Il s'écrit pour une région $r$ :

$$
G_{r}=\frac{1}{2 \bar{x}} \frac{1}{n(n-1)} \sum_{i, j}\left|x_{i r}-x_{j r}\right|, \quad \forall i, j
$$

où $n$ est le nombre de microterritoires de la région $r, i$ et $j$ sont les indices pour deux microterritoires différents et $\bar{x}$ est la moyenne des $x_{1}$ dans la région.

Tableau 2. Répartition des valeurs des quotients localisés des surfaces en $C A B$ en fonction de la période pour l'ensemble des régions

\begin{tabular}{|c|c|c|c|c|}
\hline & $<2001$ & $2001-2003$ & $2004-2006$ & $2007-2009$ \\
\hline Microterritoires avec QL > 0 (présence de CAB) & $43 \%$ & $33 \%$ & $22 \%$ & $29 \%$ \\
\hline dont microterritoires avec $Q L>1$ & $23 \%$ & $20 \%$ & $15 \%$ & $19 \%$ \\
\hline Part des QL > 1 parmi les QL > 0 & $53 \%$ & $61 \%$ & $68 \%$ & $66 \%$ \\
\hline Microterritoires avec QL = 0 (pas de CAB) & $57 \%$ & $67 \%$ & $78 \%$ & $71 \%$ \\
\hline
\end{tabular}


RECHERCHES

L'indice de Gini localisé varie entre 0 si les surfaces en $\mathrm{CAB}$ sont équi-réparties au sein de la région et 1 si les surfaces en $\mathrm{CAB}$ sont concentrées dans un seul microterritoire. Le calcul des indices de concentration a été effectué sur l'ensemble des régions et pour les quatre périodes considérées. La plage de variation de cet indice est élevée et assez restreinte $[0,7$; $0,986]$, confirmant que la conversion des surfaces en $\mathrm{AB}$ se concentre sur un nombre limité de zones au sein de chaque région. Dans toutes les régions, l'indice est relativement stable, sauf pour la période 2004-2006 qui se caractérise par un indice sensiblement supérieur, signalant une concentration plus forte des conversions, du fait comme nous venons de le voir du nombre restreint de territoires concernés.

Pour construire une typologie régionale de la dynamique de l'AB, nous avons utilisé des méthodes de classification et mobilisé plusieurs indicateurs calculés aux différentes périodes : l'indice de Gini, l'importance de l'AB dans la région (source : Agence Bio), l'intensité relative de l'effort de conversion par la contractualisation de la $\mathrm{CAB}(\mathrm{QL}$ de la région en référence à la France), ainsi que la trajectoire de croissance. Cette classification fait apparaître 5 groupes (tableau 3). En prenant comme référence la dynamique nationale, on peut ainsi distinguer des régions où l' $\mathrm{AB}$ est

Tableau 3. Typologie des régions selon la dynamique de diffusion de l'agriculture biologique

\begin{tabular}{|c|c|c|c|c|c|}
\hline & Régions & $\begin{array}{l}\text { Importance } \\
\text { de l'AB } \\
\text { (part SAU) }\end{array}$ & $\begin{array}{l}\text { Dynamique relative } \\
\text { du taux de croissance } \\
\text { des surfaces en } A B\end{array}$ & $\begin{array}{c}\text { Intensité } \\
\text { régionale } \\
\text { de la conversion } \\
\text { (QL régional) } \\
\end{array}$ & $\begin{array}{l}\text { Concentration des } \\
\text { surfaces en CAB } \\
\text { (indice de Gini) }^{12}\end{array}$ \\
\hline 1 & $\begin{array}{l}\text { Champagne-Ardenne } \\
\text { Île-de-France } \\
\text { Haute-Normandie } \\
\text { Nord-Pas-de-Calais } \\
\text { Picardie }\end{array}$ & $\begin{array}{l}\text { Très faible } \\
\text { (de 0,4 à 0,9 \% } \\
\text { de la SAU) }\end{array}$ & $\begin{array}{l}\text { Hétérogène - ten- } \\
\text { dance supérieure à } \\
\text { la moyenne nationale }\end{array}$ & $\begin{array}{l}\text { Très bas } \\
(\mathrm{QL}<0.5)\end{array}$ & $\begin{array}{l}\text { Concentration très } \\
\text { forte et stable }\end{array}$ \\
\hline 2 & $\begin{array}{l}\text { Aquitaine } \\
\text { Bourgogne } \\
\text { Centre } \\
\text { Lorraine } \\
\text { Poitou-Charentes }\end{array}$ & $\begin{array}{l}\text { Faible } \\
\text { (de } 1 \text { à 2,5 \%) }\end{array}$ & Hétérogène & $\begin{array}{l}\text { Bas } \\
(0,5<Q L<1)\end{array}$ & $\begin{array}{l}\text { Concentration } \\
\text { forte et stable }\end{array}$ \\
\hline 3 & $\begin{array}{l}\text { Auvergne } \\
\text { Bretagne } \\
\text { Basse-Normandie } \\
\text { Franche-Comté } \\
\text { Limousin }\end{array}$ & $\begin{array}{l}\text { Moyenne } \\
\text { (de 2,2 à 3,8 \%) }\end{array}$ & $\begin{array}{l}\text { Inférieure à la } \\
\text { moyenne nationale }\end{array}$ & $\begin{array}{l}\text { Élevé } \\
(1<Q L<1,3)\end{array}$ & $\begin{array}{l}\text { Concentration } \\
\text { faible et moins } \\
\text { stable }\end{array}$ \\
\hline 4 & \begin{tabular}{|l} 
Alsace \\
Rhône-Alpes \\
\end{tabular} & $\begin{array}{l}\text { Forte } \\
\text { (de } 4 \text { à 4,2\%) }\end{array}$ & $\begin{array}{l}\text { Hétérogène - ten- } \\
\text { dance supérieure }\end{array}$ & $\begin{array}{l}\text { Très élevé } \\
(\mathrm{QL} \sim 1,5)\end{array}$ & $\begin{array}{l}\text { Concentration } \\
\text { forte et stable }\end{array}$ \\
\hline 5 & $\begin{array}{l}\text { Languedoc-Roussillon } \\
\text { Midi-Pyrénées } \\
\text { PACA } \\
\text { Pays-de-la-Loire }\end{array}$ & $\begin{array}{l}\text { Forte à très forte } \\
\text { (de 3,3 à 8,7 \%) }\end{array}$ & Hétérogène & $\begin{array}{l}\text { Très élevé } \\
(1,4<Q L<1,8)\end{array}$ & $\begin{array}{l}\text { Concentration } \\
\text { faible et stable }\end{array}$ \\
\hline
\end{tabular}


plus développée, ainsi que des régions où le taux de croissance est plus soutenu.

La typologie met en avant deux situations polaires. Le groupe 1 correspond aux régions où l' $\mathrm{AB}$ est la moins développée et où la dynamique de conversion est la plus faible (la tendance supérieure du taux de croissance pouvant s'expliquer par le niveau initial très bas). L'effort de contractualisation se concentre dans quelques microterritoires, et ce quelle que soit la période considérée. Ces régions ont en commun d'être à dominante grandes cultures. À l'opposé, le groupe 5 est celui des régions les plus dynamiques, où l' $\mathrm{AB}$ est la plus présente, et où la dynamique de conversion s'est poursuivie à un rythme élevé depuis la création de la CAB. Les surfaces converties y sont moins concentrées que dans les autres régions. En comparaison avec le premier groupe, l'agriculture de ces régions est plus diversifiée.

Entre les groupes polaires, on trouve des situations intermédiaires. Le groupe 4 est très proche de par ces caractéristiques du groupe 5, mais il se différencie par une répartition des QL moins régulière sur le territoire régional. Cette particularité pourrait s'expliquer en partie par une hétérogénéité plus forte des systèmes agraires.

Le groupe 2 présente des caractéristiques proches de celles du groupe 1 , mais à des niveaux moindres; ainsi l' $\mathrm{AB}$ y est faible et la concentration de l'effort de contractualisation y est relativement forte et stable. Quant au groupe 3, il réunit les régions que l'on pourrait qualifier de « moyennes-fortes ». L'AB y est développée à un niveau égal ou supérieur à la moyenne nationale. Néanmoins, contrairement aux groupes 4 et 5 , ces régions se caractérisent par un ralentissement de la croissance de l'AB, dont le taux est inférieur au taux de croissance national. D'autre part, si l'indice de Gini montre une concentration plus faible que les groupes 1,2 et 4 , il se distingue par une plus grande instabilité, montrant que l'effort de contractualisation tend à être de plus en plus concentré entre 2000 et 2009. Ces régions se caractérisent principalement comme régions d'élevage, en particulier d'herbivores (la Bretagne diffère légèrement des autres cas, étant d'ailleurs un cas limite entre les groupes 2 et 3). La crise de l'ESB a entraîné des conversions motivées par l'effondrement des cours dans les circuits classiques pour la viande bovine; on peut penser que la crise passée, la contractualisation de nouvelles CAB a été plus localisée.

\section{Agglomération spatiale de la conversion dans les régions}

L'étape suivante de l'analyse consiste à étudier l'agglomération versus la dispersion spatiale de l'effort de conversion. Il y a agglomération si on constate une autocorrélation spatiale des zones à fort effort de conversion à l'AB. Nous mettons en œuvre un indice de Moran (Cliff et Ord, 1981). Il est calculé à partir d'une matrice de voisinage construite pour chaque région. Nous avons choisi de considérer comme voisins les microterritoires qui partagent une même frontière. La matrice de voisinage $W$ décrit le lien que possède une zone avec toutes les autres ( 1 = voisin, $0=$ sinon).

L'indice $I$ de Moran pour une région sur la base des QL des microterritoires s'écrit :

$$
I=\frac{\sum_{i} \sum_{j} w_{i j}\left(x_{i}-\bar{x}\right)\left(x_{j}-\bar{x}\right)}{\sum_{i}\left(x_{i}-\bar{x}\right)^{2}}
$$

avec les mêmes notations que précédemment et avec $w_{i j}$ un élément de la matrice de voisinage $W$. Plus la valeur de l'indice est élevée, plus forte est l'agglomération spatiale, les microterritoires avec des QL forts (supérieurs à 1) tendent à se regrouper dans l'espace régional, formant des grappes (ou clusters), de même sont regroupés ceux 
RECHERCHES

avec des QL faibles, ce qui ici nous importe moins. Un indice positif correspond à des corrélations positives (fort entouré de forts ou faible entourés de faibles) tandis qu'un indice négatif correspond à des corrélations entre voisins ayant des QL de valeur opposée (fort entouré de faibles ou faible entouré de forts). Des valeurs faibles ou négatives de $I$ correspondent donc à une dispersion (qui est aléatoire pour les valeurs proches de zéro), les microterritoires avec des QL élevés sont séparés les uns des autres.

Après standardisation des indices régionaux, seules les valeurs positives de l'indice de Moran sont significatives, et ceci plus fréquemment pour les deux premières périodes, ce qui indique une plus forte tendance à l'agglomération. Le taux de QL nuls influe sur les résultats, avec une diminution de la significativité quand ce taux augmente. Hormis pour la région RhôneAlpes pour laquelle à chaque période les conversions sont spatialement corrélées entre elles et qui a toujours l'indice le plus élevé ${ }^{13}$, il est difficile de caractériser et comparer les régions sur les différentes périodes sur la base du seul Moran ; mise à part une tendance à avoir des indices de Moran plus significatifs et plus élevés quand on compare les premiers groupes de régions ( 1 et 2 ) d'une part, le groupe 3 et enfin les groupes 4 et 5 , de l'autre.

En combinant les indices de Gini et Moran, on peut comparer le positionnement de chaque région dans le temps par rapport aux tendances moyennes. Nous avons pour cela effectué une analyse en

Figure 1.Positionnement régional 1994-2009 en fonction des indices de Gini et de Moran (selon quatre périodes)
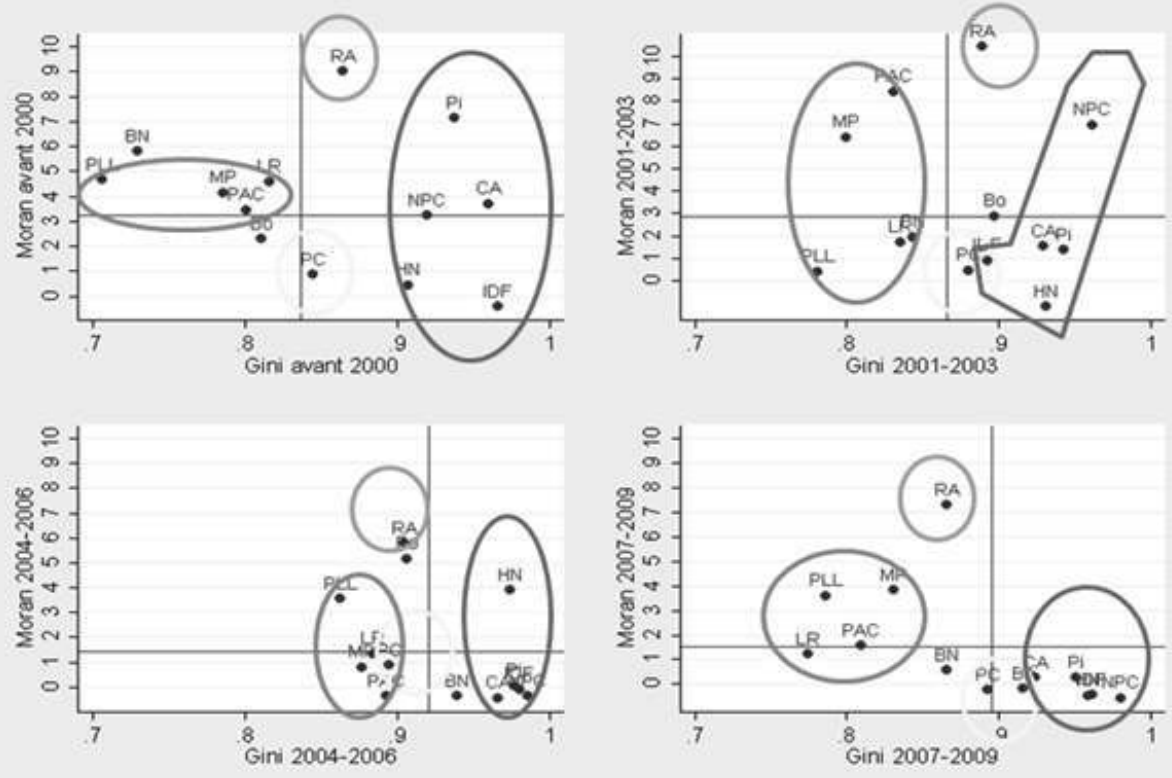

Source : les auteurs.

13. Avec une marge d'erreur de $10 \%$ (au lieu de $5 \%$ ), les régions Alsace et Lorraine ont également un indice significatif sur les 4 périodes. 
composantes principales ${ }^{14}$ pour mettre en évidence les régions qui ont les plus fortes contributions relativement aux différents indices de Gini et Moran au cours des périodes. L'ACP a fait apparaître un premier axe opposant les régions à fort ou faible Gini (concentration), mais stables sur la période. Un second axe est plutôt dédié aux indices de Moran (répartition). Aussi il est intéressant de représenter sur un même graphique ces deux indices pour les régions ayant un pouvoir explicatif significatif dans l'ACP (figure 1); ces dernières incluent les groupes polaires 1 et 5 en entier et une partie seulement des groupes intermédiaires ( 3,4 et 5).

La dimension temporelle des graphiques de la figure 1 fait apparaître une progression de l'indice moyen de concentration $^{15}$ accompagnée d'une diminution de l'indice d'agglomération (cf. les moyennes annuelles : lignes verticales et horizontales dans les graphiques). Cela reflète une diffusion de l'AB qui reste concentrée, mais touche de plus en plus de territoires. Cela s'explique par des raisons économiques (diffusion des points d'écoulement de la production), agronomiques (limites des systèmes intensifs), socioculturelles (diffusion de la sensibilité aux questions environnementales), politiques (généralisation des politiques publiques régionales ou locales). D'autre part, la période 2004-2006 et, dans une moindre mesure, la période 2007-2009 se différencient par une moindre variance des valeurs, indiquant une moindre divergence

14. L'Analyse en composantes principales (ACP) est une méthode d'analyse de données qui consiste à transformer des variables liées entre elles (corrélées) en nouvelles variables décorrélées les unes des autres, appelées «composantes principales ». Elle permet de réduire le nombre de variables et de rendre l'information moins redondante et généralement mieux interprétable.

15. Ce phénomène peut également être dû à une part plus importante de zones sans CAB au cours des 2 dernières périodes. entre régions. Une certaine stabilité temporelle du regroupement des régions selon les deux indices est visible. Par exemple, les régions Rhône-Alpes (RA) et PoitouCharentes (PC) s'opposent, la première ayant un Moran toujours très élevé, et la seconde inversement un Moran toujours plus faible que la moyenne, malgré un indice de concentration proche de celui de Rhône-Alpes. Mais pour d'autres cas, les indices (en particulier l'indice de Moran) varient selon les périodes par rapport à la moyenne, par exemple les régions MidiPyrénées (MP) ou PACA.

Les groupes polaires de la typologie établie précédemment (1, 4 et 5) présentent par construction des caractéristiques homogènes et stables du point de vue de l'indice de Gini, tandis qu'au niveau des indices de Moran, il n'y a que peu de cohérence et de stabilité au sein des groupes, même si les indices pour les groupes 5 et 4 sont tendanciellement supérieurs à ceux des autres groupes, en particulier du groupe 1. La faible significativité des indices et leur inconstance limitent les interprétations. Néanmoins, si on compare les régions une à une, on peut dégager certains enseignements. Par exemple, Rhône-Alpes et Pays-de-la-Loire occupent dans les 4 périodes des positions opposées. Si ces deux régions peuvent être qualifiées de régions fortes en $\mathrm{AB}$ (part de la SAU supérieure à la moyenne, dynamisme des conversions), sa diffusion a emprunté dans chacune une forme différente : une répartition de l'effort de conversion plus concentrée et agglomérée (en grappes) dans le cas de Rhône-Alpes versus une répartition plus diffuse dans les Pays-de-la-Loire. Un autre exemple est celui de la Basse-Normandie, qui dans les deux premières périodes a des caractéristiques proches de celles du groupe 5, avant de s'apparenter plus au groupe 1 lors des périodes plus récentes, avec un ralentissement de la croissance de l'AB dans cette région. 
Les indices de Gini et de Moran ont donné une vision globale sur la concentration et l'agglomération de l'effort de conversion en $\mathrm{AB}$ dans chaque région. Il se peut cependant que des configurations d'agglomération spécifiques soient présentes dans les régions. La question est alors de savoir plus en détail comment se distribuent spatialement ces agglomérations. Nous allons pour cela étudier les dynamiques infrarégionales et analyser les types d'agglomération via le calcul et la représentation cartographique d'indices locaux.

\section{Typologie des formes d'agglomération : associations spatiales}

Des types locaux d'agglomération de l'effort de conversion vont être maintenant mis en évidence au sein des régions. Nous étudions les associations locales (Oliveau, 2010) de valeur des QL calculés au niveau des microterritoires avec ceux des territoires voisins, en se basant sur les statistiques de LISA (Local Indicator of Spatial Agglomeration) (Anselin, 1995) et à l'aide de la méthode du nuage de points de Moran (Moran scatterplot). Le nuage de points de Moran permet d'étudier la ressemblance de chaque individu avec ses voisins par rapport à l'ensemble de l'échantillon. Il divise notre population en 4 classes : des zones avec des valeurs de QL élevées/ faibles entourées de zones avec également de forts/faibles QL (notées HH/LL) ; des zones avec des valeurs de QL élevées/ faibles entourées de zones avec des QL faibles/forts (notées HL/LH).

La statistique de LISA, pour les microterritoires d'une région donnée, est construite de la façon suivante (Anselin 1995) :

$$
I_{i}=\frac{n\left(x_{i}-\bar{x}\right) \sum_{j} w_{i j}\left(x_{j}-\bar{x}\right)}{\sum_{i}\left(x_{i}-\bar{x}\right)^{2}}
$$

Un $I_{i}$ positif indiquera des associations spatiales de microterritoires avec un QL similaires (HH ou LL), tandis qu'un $I_{i}$ négatif mettra en évidence des associations spatiales de microterritoires avec un QL différents (HL ou LH) encore appelés spatial outliers. Tester la significativité de ces indices demande de connaître la distribution des LISA sous l'hypothèse nulle d'indépendance spatiale ${ }^{16}$. Concrètement les valeurs non significatives des LISA correspondent à des individus qui ont un QL similaire à celui de leur voisin et proche de la moyenne de l'échantillon. L'étape suivante consiste à cartographier les territoires pour mettre en évidence les zones de corrélations spatiales significatives de territoires en forte (resp. faible) conversion (HH, resp. LL) ou bien des territoires plus contrastés (HL ou LH) où des agglomérations significatives se produisent entre microterritoires hétérogènes (cartes 2 à 5 en annexe). Lorsque dominent dans une région les configurations de ce dernier type, l'effort de conversion est globalement plus réparti que dans le cas inverse ${ }^{17}$.

La classe LL est la plus représentée, du fait d'un nombre important de microterritoires sans surface aidée en conversion; de ce fait la significativité est faible pour cette classe (résultat similaire à celui de Guillain et Le Gallo, 2010). Les zones HH, où se produit l'agglomération, sont peu représentées ; elles le sont mieux si l'on s'intéresse aux seules valeurs significatives de la

16. Nous utilisons pour cela le logiciel Geoda (Anselin, 2005) qui procède par simulation de Monte-Carlo pour effectuer les tests de significativité.

17. Bien que ce ne soit pas l'objet de ce papier, on peut toutefois noter ici que les résultats obtenus sont sensibles à l'échelle géographique élémentaire (unités d'analyse) et à la définition du voisinage ; il s'agit du problème dit des unités spatiales modifiables (modifiable areal unit problem - MAUP, Openshaw, 1984). Pour une discussion des conséquences de ces choix, voir Oliveau (2010). 
statistique de LISA. Elles sont plus représentées que les territoires HL qui sont des microterritoires isolés où se concentre l'effort de conversion. On trouve logiquement une participation de plus en plus élevée des zones $\mathrm{HH}$ et HL quand on passe des groupes 1 à 5 de la typologie des régions. La statistique de LISA permet d'expliciter spatialement l'analyse faite à partir des indices globaux.

Nous nous intéresserons maintenant aux formes d'agglomération rencontrées dans les 6 régions des groupes 4 et 5 , qui sont les régions où l' $\mathrm{AB}$ est la plus développée, et où les indicateurs tant globaux que locaux sont les plus significatifs. Nous associerons les formes de concentration et d'agglomération de l'effort de contractualisation en $\mathrm{CAB}$ et les dynamiques régionales de développement de l'AB.

D'un point de vue général, on note la diminution de l'agglomération dans le temps. En effet, la proportion de zones $\mathrm{HH}$ diminue entre 2001 et 2009. Une tendance de fond se dessine donc, indiquant que la diffusion de l'AB est passée d'un développement centré sur quelques clusters à une croissance spatialement plus diffuse. Cette tendance est commune à l'ensemble des régions, même si les dynamiques peuvent diverger d'une région à l'autre et selon les périodes. Corrélativement, la fréquence des classes indiquant une plus grande mixité des valeurs (HL et LH) augmente entre la première et la dernière période.

L'évolution des formes de diffusion suggère une «normalisation » de l'AB recouvrant une modification des facteurs et des conditions d'adoption. L'agriculture biologique s'est initialement construite en grande partie en opposition à la « modernisation »des structures et pratiques agricoles et donc à travers des réseaux à l'écart du système professionnel dominant (Sylvander, 1997). Les politiques publiques accordant une forte attention au développement de l'AB, l'INRA et les
Chambres d'agriculture participant avec les réseaux spécialisés aux dynamiques innovantes en $\mathrm{AB}$, sa diffusion passe moins par des formes de proximités socioculturelles et géographiques, mais devient une démarche plus individuelle et basée sur une opportunité économique (sans être l'unique motivation), en même temps que s'élargissent les circuits de collecte AB et que se structurent les filières. Cette évolution ne supprime toutefois pas l'hétérogénéité spatiale.

Les zones concernées par des phénomènes d'agglomération présentent certaines caractéristiques communes. Elles se situent souvent dans des zones de moyennes montagnes (Vosges, Vercors, Diois, Ardennes, Corbières, Morvan...) et/ou dans des zones orientées vers des systèmes herbagers (bocage normand, nord de la région nantaise...). Les zones «montagne » se caractérisent par une proportion plus importante de microterritoires de classe $\mathrm{HH}$ que les zones « défavorisées », qui en ont elles-mêmes une proportion plus forte que les zones « plaine». Ce phénomène se retrouve sur les quatre périodes et est encore plus marqué si on ne regarde que les Moran locaux significatifs. Toutefois, il s'amenuise dans le temps, la différence avec les zones «plaine » se réduisant.

La Région Rhône-Alpes est celle où les indicateurs globaux et locaux d'agglomération sont les plus élevés, avec les clusters les plus importants et les plus significatifs. C'est le cas pour la partie sud-est de la région (le sud de l'Isère et l'est de la Drôme), où on retrouve une grappe importante de $\mathrm{HH}$ pour les 4 périodes. Lamine et al. (2011) ont étudié la formation et la dynamique de l'AB dans ce territoire, qui s'appuie sur des formes de coordination locales. Cependant la taille de ce cluster tend à diminuer dans le temps, et l'agglomération y est plus faible : les microterritoires de classe $\mathrm{HH}$ sont plus répartis dans 
la région, formant des clusters de moindre envergure. Parallèlement, on retrouve plus de microterritoires de classe mixte (HL et LH), signe d'une répartition plus aléatoire des zones avec un QL élevé.

En Alsace, l'indice de Moran global est moins élevé, mais il est toujours significatif à 10 ou à $5 \%$. On lit très clairement au niveau de la carte la différence entre les zones de plaine et celles des reliefs vosgiens et de l'Alsace bossue ${ }^{18}$ où se concentrent les microterritoires de classe HH. Néanmoins, comme dans le cas précédent, on constate une diminution progressive de ce phénomène d'agglomération, même s'il persiste en Alsace bossue.

Languedoc-Roussillon présente, contrairement à Rhône-Alpes, un faible niveau d'agglomération, tant au regard de l'indice de Moran global que des indices locaux. Néanmoins il partage la même décroissance dans l'agglomération : alors que dans la première période il existe une grappe importante de $\mathrm{HH}$ au niveau des Corbières (que l'on retrouve dans une moindre mesure aux deux périodes sui-

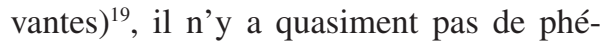
nomène d'agglomération repérable pour 2007-2009. On a au contraire une répartition plus homogène des classes HH et HL, et la seule agglomération identifiable est en fait liée à une zone où l'effort est faible (en Nord-Lozère). Dans cette région, les conversions concernent - principalement dans la période récente - des surfaces en vigne et compte tenu de l'importance de la vente directe, la répartition des conversions limite la concurrence entre producteurs sur les marchés locaux et les sites touristiques.

18. Région naturelle d'Alsace, tournée principalement vers l'élevage bovin (lait). Voir Hellec et Blouet (2011) pour une présentation du développement de l'AB dans cette région.

19. Sans doute liée au fait que certaines caves coopératives de villages se sont converties totalement ou majoritairement à la production de raisins en agriculture biologique, à la fin des années 1990.
En PACA, région leader pour la part de la SAU dédiée à l' $\mathrm{AB}$, les cartes et indices montrent que les clusters identifiés au cours de la première et encore plus de la deuxième période ne se retrouvent plus les années suivantes. On retrouve quelques zones d'agglomération, mais plus réduites et moins significatives.

En Midi-Pyrénées, la situation diffère, le niveau d'agglomération se maintient, l'indice de Moran global redevenant même significatif pour la dernière période, contrairement aux deux précédentes ${ }^{20}$. Deux principales grappes ressortent : dans le Sud-Aveyron (dans la région touristique des Grands Causses, territoire d'un Parc naturel régional) et dans une moindre mesure au nord de l'Ariège (à proximité de la métropole toulousaine). Si, par ailleurs, on trouve plus de petits clusters en 2007-2009 par rapport à la première période, on peut noter également que dans la même période le département des Hautes-Pyrénées se distingue puisqu'il ne comprend que des microterritoires de classe LL.

Dans le cas des Pays-de-la-Loire, les cartes représentant les classes de Moran montrent une plus forte dispersion de l'effort de conversion dans la dernière période. Cependant, les indices globaux et locaux significatifs mettent en avant le maintien d'un certain niveau d'agglomération, que l'on retrouve en particulier avec le cluster au nord de Nantes, qui regroupe quasiment l'ensemble des microterritoires de classe HH significatifs.

On peut ici souligner deux formes de concentration différentes selon les régions et les périodes considérées, avec une agglomération par grappes concentrées d'un côté, et d'un autre côté une répartition plus diffuse, non seulement entre microterritoires, mais aussi avec une meilleure

20. Il est à noter par ailleurs que Midi-Pyrénées est une des régions qui a connu une des plus fortes progressions de la $\mathrm{SAU}$ en $\mathrm{AB}$ ces dernières années. 
répartition géographique des zones avec un taux relatif de surface en CAB élevé. Audelà de la comparaison des régions à un instant $t$, il est également possible de mobiliser des outils de l'analyse spatiale pour comparer les évolutions dans le temps.

\section{Dépendances spatio-temporelles de l'effort de conversion}

Les sections précédentes ont mis en évidence le fait que des changements apparaissent au cours du temps à la fois dans la concentration et dans l'agglomération de l'effort de conversion en bio. On peut enrichir cette analyse en testant la dépendance temporelle des effets d'agglomération ${ }^{21}$ (que l'on appelle phénomène de contagion), pouvant provenir d'effets d'apprentissage collectif de la part des agriculteurs ou de l'appareil local de conseil ou d'autres interdépendances spatio-temporelles, en particulier liées aux systèmes de production territoriaux et à la localisation des marchés. La statistique relative aux dates $t$ et t-k s'écrit :

$S T I_{k}=\frac{\sum_{i, j} w_{i j}\left(x_{i, t-k}-\bar{x}_{t-k}\right)\left(x_{j, t}-\bar{x}_{t}\right)}{\sqrt{\sum_{i}\left(x_{i, t-k}-\bar{x}_{t-k}\right)^{2}} \sqrt{\sum_{i}\left(x_{i, t}-\bar{x}_{t}\right)^{2}}}$

Notons que pour $k=0$ cette statistique coïncide avec la statistique de Moran définie plus haut. Nous considérons ici l'éventualité d'une dépendance temporelle (entre périodes) d'ordre $1(k=1)$ et nous calculons les Moran spatio-temporel globaux, d'une période à l'autre, pour les 6 régions les plus significatives retenues dans notre analyse. Les résultats sont présentés dans le tableau 4.

Les indices de Moran multivariés sont tous positifs, indiquant une possible contagion. Cependant ils sont très faibles et parfois non significatifs, excepté pour la Région Rhône-Alpes où les trois indices de Moran sont de l'ordre de 0.15 et sont fortement significatifs.

À partir de la représentation cartographique (figure 2), la dynamique de contagion en Rhône-Alpes peut être analysée. D'une part, la contagion «positive » (de type $\mathrm{HH}$ ) se concentre dans un département, la Drôme, et plus particulièrement dans sa partie orientale. On peut ainsi relier l'existence et la permanence de ce cluster à un phénomène de contagion, où un microterritoire fort à une période tend à attirer des voisins forts à la période suivante. Le Moran multivarié met ainsi en avant les clusters qui se maintiennent d'une période à l'autre. Il vient donc confirmer que l'agglomération mise en évidence par le Moran univarié s'inscrit dans des dynamiques temporelles.

En comparant les cartes pour les différentes régions, on peut noter deux autres éléments. D'abord l'autocorrélation spatio-temporelle tend à diminuer dans le temps ; les déterminants spatiaux sont donc moins significatifs au cours des périodes plus récentes par rapport à la dynamique

Tableau 4. Moran multivariés (STI)

\begin{tabular}{lccc}
\hline \multicolumn{1}{c}{ Régions } & 2000 vs. 2003 & 2003 vs. 2006 & 2006 vs. 2009 \\
\hline Pays-de-la-Loire & $0.067^{*}$ & $0.082^{*}$ & $0.074^{*}$ \\
Midi-Pyrénées & $0.096^{* * *}$ & $0.061 *$ & $0.043^{*}$ \\
Rhône-Alpes & $0.146 * * *$ & $0.148 * * *$ & $0.059 * *$ \\
Languedoc-Roussillon & $0.079 *$ & 0.053 & 0.017 \\
Provence-Alpes-Côte-D'azur & 0.013 & 0.035 & -0.027 \\
Alsace & $0.122^{* *}$ & $0.157 * * *$ & 0.012
\end{tabular}

21. Wartenberg (1985) introduit la notion de Moran spatio-temporel (STI).

Note : Significativité à : *** $1 \%$ * $5 \%$ * $10 \%$. Source : les auteurs. 
2000 vs. 2003

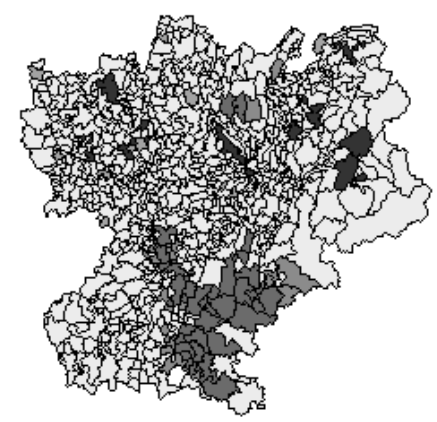

2006 vs. 2009

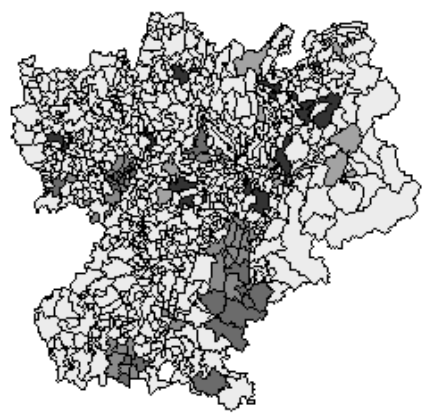

initiale. Ensuite, si les microterritoires de classe HH sont particulièrement regroupés entre les périodes 2000 et 2003, ils sont de plus en plus répartis sur l'ensemble du territoire au fur et à mesure qu'on avance dans le temps.

Dans la Région Pays-de-la-Loire on note également le maintien voir le renforcement dans le temps du principal cluster, au nord de Nantes. À l'inverse, dans une région comme PACA, les zones $\mathrm{HH}$ varient avec le temps et sont en plus mieux réparties. Le développement de l'AB y paraît donc moins inscrit dans une dépendance temporelle, ce qui se retrouve à la fois dans les indices locaux (Moran multivariés significatifs) et globaux (Moran multivariés par période pour la région). Un troisième cas de figure est celui de Midi-Pyrénées, où il existe bel et bien des grappes de microterritoires de type $\mathrm{HH}$, mais qui varient d'une
2003 vs. 2006

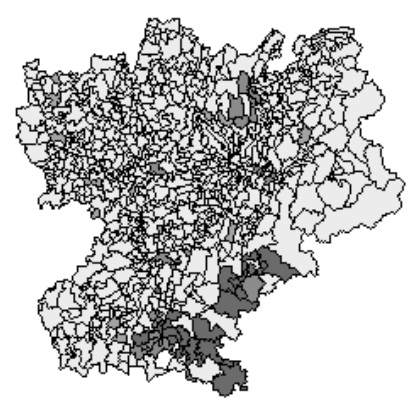

Légende

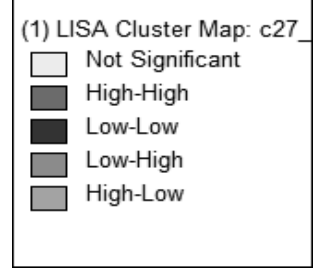

Source : les auteurs.

période à l'autre (le Nord-Ariège entre la période avant 2000 et 2001-2003, et le Sud-Aveyron entre les périodes 2004-2006 et 2007-2009).

\section{Discussion}

Les effets d'autocorrélation spatiale et/ou temporelle viennent appuyer l'hypothèse de l'existence de rendements croissants d'adoption dans le cas de l'AB (Pernin, 1994) $)^{22}$. Ils peuvent être liés en particulier à

22. Dans ce contexte, le bénéfice (monétaire ou non) que chaque producteur adoptant peut attendre est d'autant plus grand que d'autres adoptent, ce qui explique ce que nous appelons la contagion. Le risque économique que prennent les derniers adoptants est plus faible que pour les premiers, ce qui stimule l'adoption. Évidemment ce phénomène a une limite. Le même raisonnement peut être tenu concernant les politiques publiques de soutien. 
des effets de seuil (nombre minimum d'exploitations pour rentabiliser une nouvelle route de collecte ou un atelier de transformation bio, etc.) et à des effets de proximité (mimétisme, réseaux locaux d'échange, etc.). À ces déterminants de la dépendance entre les conversions individuelles, s'ajoute une efficacité institutionnelle croissante avec l'expérience collective accumulée au niveau territorial. On peut donc parler de path dependence spatiale, comme cela a été montré dans d'autres études (Bichler $e t$ al., 2005). Toutefois, on note une tendance à une diminution de ce phénomène dans la diffusion de l'AB.

En reprenant l'analyse de Pernin (1994), on peut interpréter cette tendance comme le dépassement d'une phase plus territorialisée, plus localisée des rendements croissants d'adoption ; en d'autres termes par un changement d'échelle de ces rendements, qui va de pair avec l'organisation des filières. La nature et la spatialité des sources de rendements croissants ont évolué, des seuils ont été franchis, qui font que la géographie de la conversion est devenue plus diffuse et moins concentrée qu' auparavant. Il faudrait toutefois pouvoir prendre également en compte le stock, c'est-à-dire la part de surface qui reste à convertir ou plus précisément qui pourrait être convertie, car il pourrait y avoir des phénomènes de saturation. Cependant, les résultats présentés ici montrent que les territoires où l' $A B$ est la plus avancée ne semblent pas saturés et sont au contraire souvent encore les plus dynamiques. De plus, la diminution des indices d'agglomération ne signifie pas la disparition de ce phénomène ; la diffusion de l'AB conserve une distribution spatiale hétérogène, ce qui ressort clairement dans les différences observées inter- et intra-régionales. Enfin, au niveau des dispositifs publics de soutien, il apparaît clairement une particularité de la période des CAD (2004-2006) avec une moindre dynamique de contractualisation, et une concentration plus forte dans les zones les plus avancées en termes de développement de l'AB (phénomène en partie lié à l'agrandissement des exploitations déjà en bio).

$$
\begin{gathered}
* \\
* *
\end{gathered}
$$

À travers la mobilisation d'une série de données administratives sur l'agriculture biologique et les aides à la conversion (CAB), la dynamique de la diffusion de la conversion à l' $\mathrm{AB}$ a pu être caractérisée dans sa dimension spatiale et temporelle. Quatre angles de la dynamique de la conversion en $\mathrm{AB}$ ont pu être approchés : l'intensité ou l'effort relatif de contractualisation au niveau de microterritoires, avec le QL ; la concentration de cet effort (indices de Gini régionaux, par périodes), son agglomération (indices de Moran régionaux et locaux - LISA) et la contagion (indices de Moran spatio-temporels). Différentes dynamiques et configurations spatiales régionales, mais également infrarégionales ont été mises en lumière. Cette hétérogénéité spatiale reflète tant la structuration économique de l'AB que l'hétérogénéité régionale, départementale et locale des dispositifs de soutien, plus ou moins incitatifs et efficaces.

Dans un premier temps, nous avons proposé une typologie des régions en fonction d'indices globaux sur l'intensité et la concentration de l'effort de conversion et aussi en fonction de la diffusion de l'AB. En nous intéressant ensuite au niveau infrarégional, nous avons mis en évidence différentes formes d'agglomération au sein des régions. Ainsi, des régions où le développement de l'AB est important peuvent se caractériser par des regroupements en grappes (ou clusters) de microterritoires à QL élevé, ou au contraire ces derniers peuvent être répartis sur l'ensemble du territoire. Par exemple, la région RhôneAlpes se distingue par ses indices globaux et locaux plus élevés, témoignant de phénomènes d'agglomération et de contagion plus importants que d'autres régions avec 
RECHERCHES

des niveaux de concentration similaires. Cependant, comme dans la plupart des régions, la tendance générale est à la diminution de l'intensité de ces phénomènes, témoignant d'une plus large diffusion de l'AB.

Du fait même de la multiplicité des échelles spatiales et temporelles, ainsi que de l'idiosyncrasie régionale et locale, l'interprétation des données est souvent limitée. Des approches plus qualitatives et systémiques, comme celle de Lamine et al. (2011) sur les dynamiques territoriales de transition vers l'AB, pourraient apporter des éléments complémentaires. C'est avant tout dans une posture exploratoire à la fois descriptive et méthodologique que s'inscrit ce travail, qui ouvre néanmoins une série de pistes de recherche pour analyser les mécanismes en jeu dans la diffusion de l'agriculture biologique, en fonction de facteurs individuels, collectifs et territoriaux.

La présente recherche a été conduite avec le soutien de l'ANR (programme SYSTERRA) dans le cadre du projet DynRurABio, regroupant plusieurs équipes de I'INRA et du CNRS. Les auteurs remercient les deux relecteurs anonymes de la revue, dont les remarques ont permis d'améliorer la présentation des résultats de ce travail.

\section{RÉFÉRENCES BIBLIOGRAPHIQUES}

Agence Bio (2012). L'agriculture biologique française : les chiffres clés. Édition 2012 (Chiffres 2011), 260 p.

AND-I (2008). Évaluation ex post du Plan de Développement Rural National. Soutien à l'agroenvironnement. Annexe : Étude de cas sur l'agriculture biologique, 99 p. (disponible sur le site du ministère de l'Agriculture).

Anselin L. (1995). Local indicators of spatial association - LISA. Geographical Analysis, 27: 93-115.

Anselin L. (2005). Exploring spatial data with GeoDa. A Workbook, available at http:// geodacenter.asu.edu/system/files/geodaworkbook.pdf

Beauchesne A., Bryant C. (1999). Agriculture and Innovation in the Urban Fringe: The Case of Organic Farming in Quebec, Canada. Tijdschrift voor Economische en Sociale Geografie, vol. 90, n 3, p. 320-328.

Bichler B., Häring A. M., Dabbert S., Lippert C. (2005). Determinants of spatial distribution of organic farming in Germany. Paper at: Researching sustainable systems, Adelaide (Australia), 21-23 sept.

Cliff, A., Ord J. K. (1981). Spatial processes: Models and applications. London: Pion.
Frederiksen P., Langer V. (2004). Localisation and concentration of organic farming in the 1990s - the Danish case. Tijdschrift voor Economische en Sociale Geografie, vol. 95, $\mathrm{n}^{\circ} 5$, p. 539-549.

Gabriel D., Carver S. J., Durham H., Kunin W. E., Palmer R. C., Sait S. M., Stagl S., Benton T. G. (2009).The spatial aggregation of organic farming in England and its underlying environmental correlates. Journal of Applied Ecology, $\mathrm{n}^{\circ} 46$, p. 323-333.

Géniaux G., Lambert, M., Bellon S. (2009). Analyse de la diffusion spatiale de l'agriculture biologique en région Provence-AlpesCôte d'Azur (Paca) : construction d'une méthodologie d'observation et de prospective. Innovations Agronomiques, $\mathrm{n}^{\circ} 4$, p. 417-426.

Géniaux G., Latruffe L., Lepoutre J., Mzoughi N., Napoléone C., Nauges C., Sainte-Beuve J., Sauterau N. (2010). Les déterminants de la conversion à l'agriculture biologique : une revue de la littérature économique. INRA Agribio3, Projets PEPP et EPAB. http://www.inra.fr/comite_agriculture_biologique/content/download/3327/33475/version/6/file/Livrable_1_ EPAB_PEPP_final.pdf. 


\section{Approche spatiale de la conversion à l'agriculture biologique}

Guillain R., Gallo J. L. (2010). Agglomeration and dispersion of economic activities in and around Paris: an exploratory spatial data analysis. Environment and Planning B: Planning and Design, vol. 37, $\mathrm{n}^{\circ} 6$, p. 961-981.

Guilmoto C. Z. (2008). L'inscription spatiale de la discrimination de genre en Inde. Effets des distances sociale et géographique. L'Espace géographique, vol. $35, \mathrm{n}^{\circ} 1, \mathrm{p} .1-15$

Hellec F., Blouet A. (2011). L'essor de l'élevage laitier biologique en Alsace Bossue. In Van Dam D., Streith M., Nizet J. (dir.), Le bio en devenir. Le cas alsacien. Bruxelles, Peter Lang, p. 79-91.

Ilbery B., Holloway L., Arber R. (1999).The geography of organic farming in England and Wales in the 1990s. Tijdschrift voor Economische en SocialeGeografie, vol. 90, $\mathrm{n}^{\circ} 3$, p. 285-95.

Ilbery B., Maye D. (2011). Clustering and the spatial distribution of organic farming in England and Wales. AREA Royal Geographical Society, vol. 43, n 1, p. 31-41.

Lamine C., Landel P. A., Duffaud-Prevost M. L. (2011). Dynamiques territoriales de transition vers l'agriculture biologique. Colloque SFER «Les transversalités de l'agriculture biologique ». 23 et 24 juin, Université de Strasbourg.

Lewis D., Barham B., Robinson B. (2011). Are there spatial spillovers in the adoption of clean technology? The case of organic dairy farming. Land Economics, vol. 87, $\mathrm{n}^{\circ} 2$, p. 250-267.

Nyblom J., Borgatti S., Roslakka J., Salo M. A. (2003). Statistical analysis of network dataan application to diffusion of innovation. Social Networks, $\mathrm{n}^{\circ} 25$, p. 175-195.

Oliveau S. (2010). Autocorrélation spatiale : leçon du changement d'échelle. L'Espace géographique, vol. 39, $\mathrm{n}^{\circ}$ 1, p. 51-64.

Openshaw S. (1984). The modifiable areal unit problem. Concepts and Techniques in Modern Geography, n 38, 41 p.

Pernin J.-L. (1994). Réseaux et rendements croissants d'adoption dans l'agriculture biologique en France. Revue d'économie industrielle, vol. 70, $\mathrm{n}^{\circ} 4$, p. 49-71.

Quelin C. (2010). Agriculture biologique : la fin du retard français? Les Études de l'ASP, $14 \mathrm{p}$.

Risgaard M. L., Frederiksen P., Kaltoft P. (2007). Socio-cultural process behind the differential distribution of organic farming in Denmark. Agriculture and Human Values, vol. 24, n 4, p. 445-459.

Sylvander B. (1997). Le rôle de la certification dans les changements de régime de coordination : l'agriculture biologique, du réseau à l'industrie. Revue d'économie industrielle, $\mathrm{n}^{\circ} 80$, p. 47-66.

Wartenberg D. (1985). Multivariate spatial correlation - a method for exploratory geographical analysis. Geogaphical Analysis, $\mathrm{n}^{\circ} 17$, p. $263-283$. 


\section{ANNEXES}

Carte 1. Quotients localisés des c27 pour les surfaces en CAB par rapport à la région en 2007-2009
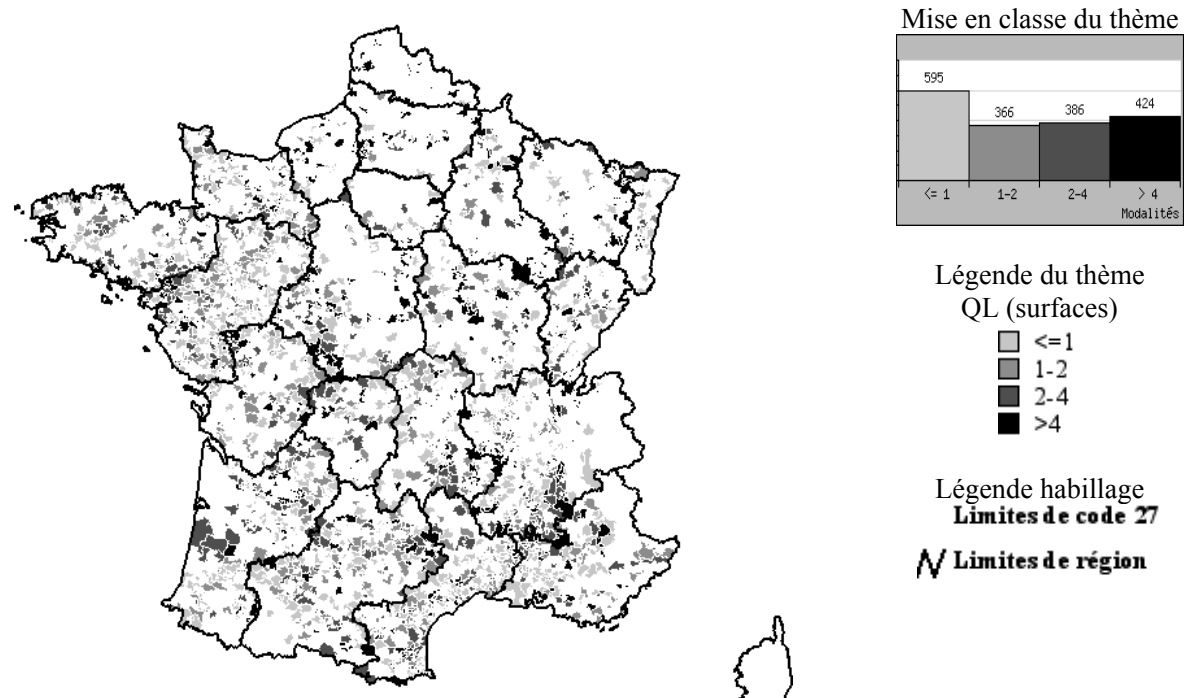

Légende du thème QL (surfaces)

$\square<=1$

$\square 1-2$

D $2-4$

Légende habillage

Limites de code 27

$N$ Limites de région

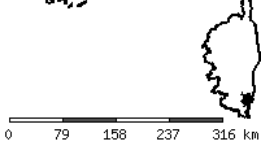

Source: ASP.

Carte 2. Carte des Moran scatterplot pour les surfaces en CAB avant 2001
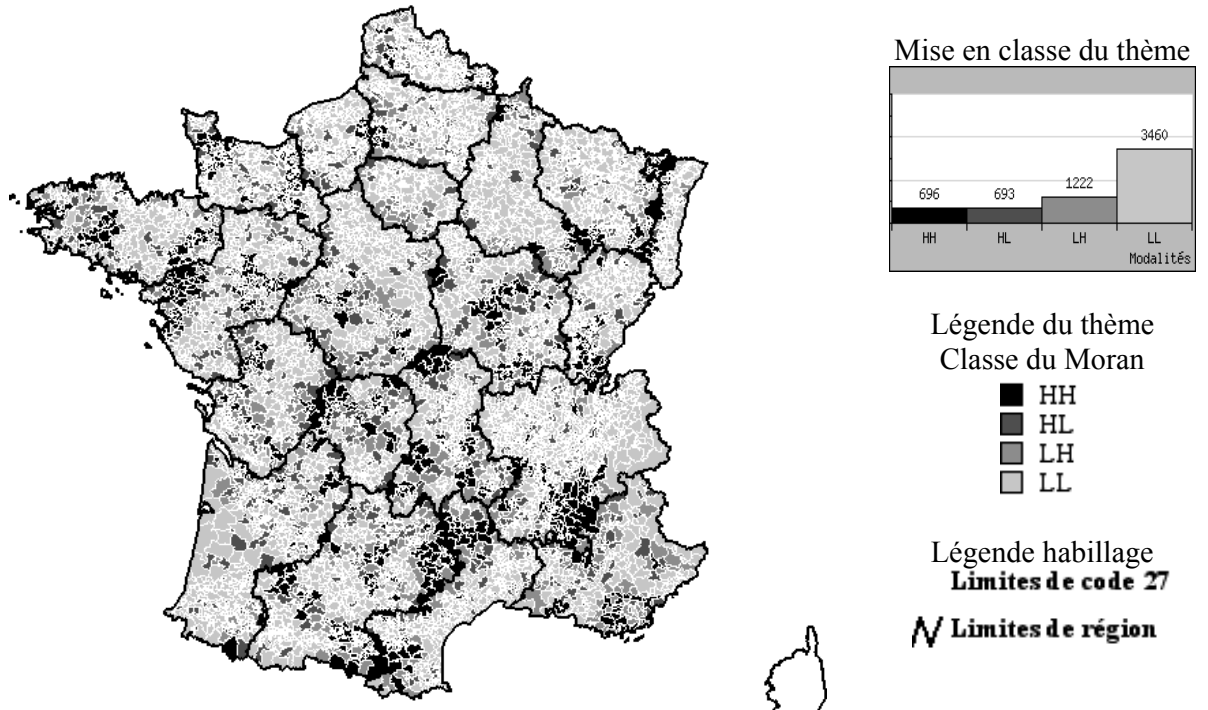

Légende du thème Classe du Moran
$\square \mathrm{HH}$
$\square \mathrm{HL}$
$\square \mathrm{LH}$

Légende habillage Limites de code 27

$N$ Limites de région

Source : $A S P-C D R$. 
Approche spatiale de la conversion à l'agriculture biologique

Carte 3. Carte des Moran scatterplot pour les surfaces en CAB pour 2007-2009
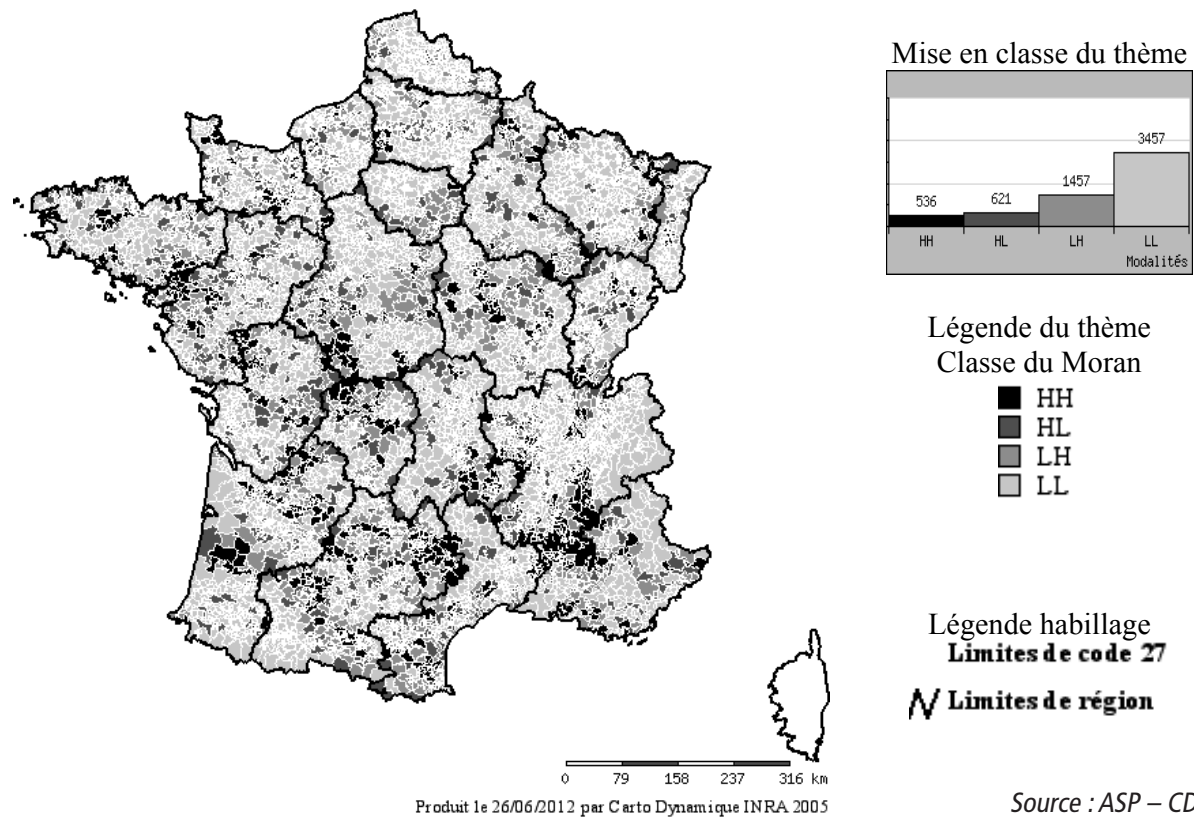

Légende du thème Classe du Moran

$\square \mathrm{HH}$
$\square \mathrm{HL}$
$\square \mathrm{LL}$

Légende habillage Limites de code 27

$N$ Limites de région

Source : $A S P-C D R$.

Carte 4. Carte des Moran scatterplot significatifs pour les surfaces en CAB avant 2001 dans les 6 régions étudiées

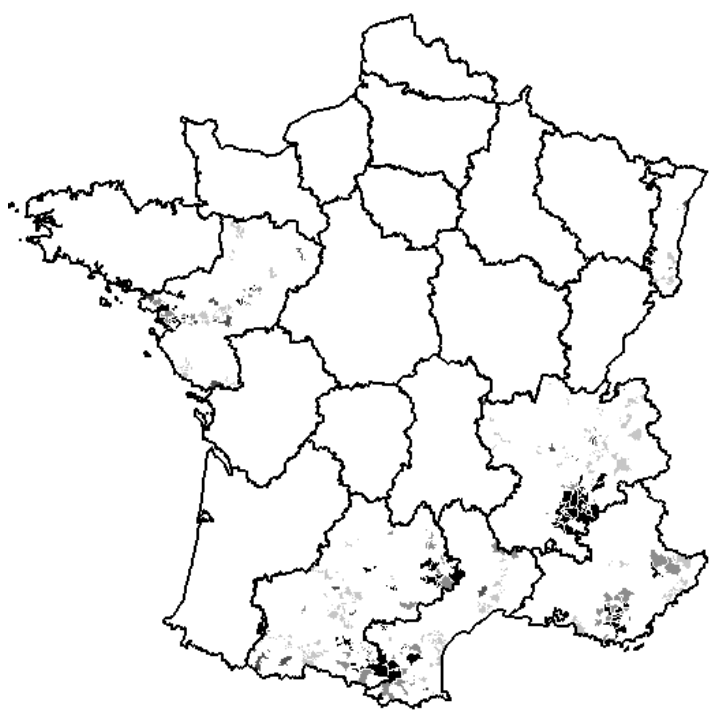

$\{\underbrace{}_{316 \mathrm{~km}}\}$

Produit 1e 26/06/2012 par C arto Dymami ique INRA 2005
Mise en classe du thème

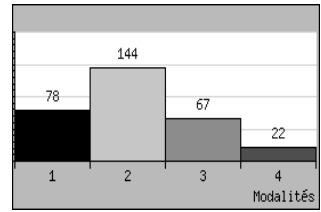

Légende du thème lisa 9100

$\square \mathrm{HH}$

$\square \mathrm{HL}$

$\square \mathrm{LH}$

$\square \mathrm{LL}$

Légende habillage Limites de code 27

$N$ Limites de région

Source : $A S P-C D R$. 
RECHERCHES

Gilles ALLAIRE, Éric CAHUZAC, Thomas POMÉON, Michel SIMIONI

Carte 5. Carte des Moran scatterplot significatifs pour les surfaces en CAB pour 2007-2009 dans les 6 régions étudiées

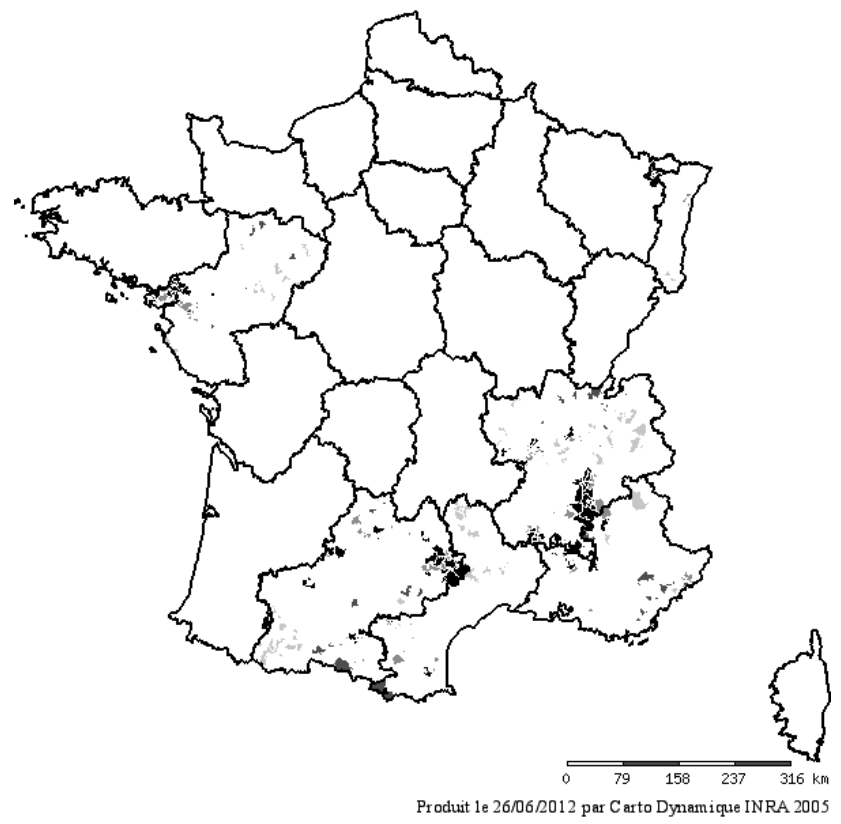

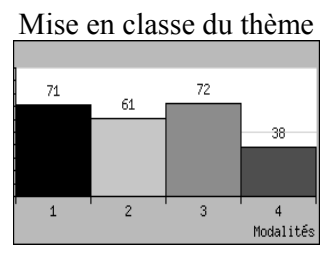

Légende du thème lisa_5reg_09

HH

$\square \mathrm{HL}$

$\square \mathrm{LH}$

$\square \mathrm{LL}$

\section{Légende habillage Limites de code 27 \\ $N$ Limites de région}

Source : $A S P-C D R$. 